\title{
Drift motion of ionospheric arc-like absorption regions observed with a 256-beam imaging riometer in Alaska
}

\author{
Shoji Kainuma ${ }^{1}$, Mamoru Ishii ${ }^{2}$, Yasuhiro Murayama ${ }^{2}$, Takashi Kikuchi ${ }^{2}$, Hirotaka Mori ${ }^{2}$, and Kiyoshi Igarashi ${ }^{2}$ \\ ${ }^{1}$ Yamagawa Radio Observatory, 2719 Narikawa, Yamagawa-machi, Ibusuki-gun, Kagoshima 891-0516, Japan \\ ${ }^{2}$ Communication Research Laboratory, Ministry of Posts and Telecommunications, 4-2-1 Nukui-Kitamachi, Koganei-shi, Tokyo 184-8795, Japan
}

(Received June 11, 1999; Revised April 19, 2001; Accepted April 19, 2001)

\begin{abstract}
We studied the local time dependence of the direction of cosmic noise absorption drift using a large data set obtained with a $16 \times 16$-antenna imaging riometer in Poker Flat, Alaska $\left(65.1^{\circ} \mathrm{N}, 147.5^{\circ} \mathrm{W}\right.$ in geographic coordinates; $65.4^{\circ} \mathrm{N}$, $100.7^{\circ}$ in geomagnetic coordinates). The drift velocities were determined using regression lines for the absorption peaks, which were automatically detected. Based on the characteristics of the motion of the drift region, the events were categorized into three magnetic local time (MLT) groups: I (13-19 MLT), II (21-01 MLT) and III (01-07 MLT). Most events in group I move southwestward with steady velocities. Events in group II had various velocities and directions. Events in group III also had various velocities, but the tilt angles of their vector velocities from the eastward axis were between $-90^{\circ}$ and $90^{\circ}$, meaning that they moved eastward. These results are consistent with some found in previous studies. A polar plot of the drift had a pattern with features similar to that of magnetospheric convection, which has been reported on several studies: equatorward in the pre-midnight sector and sunward in the morning and evening sectors.
\end{abstract}

\section{Introduction}

Although the structures in which most auroral radio absorption occurs are complicated, some absorption events have relatively simple structures. The 'spiky events', 'bays', and 'peaks' are examples of such events, and have been thoroughly studied (Hargreaves, 1967; Pudovkin et al., 1968; Jelly, 1970; Theander, 1972; Berkey et al., 1974; Olson et al., 1980). Hargreaves (1970) measured the velocity of motion of small-scale absorption regions using oblique riometer antennas with ionospheric projections that were separated by over $250 \mathrm{~km}$. He obtained velocities between $80 \mathrm{~m} / \mathrm{s}$ and $3.3 \mathrm{~km} / \mathrm{s}$ with directions eastward in the morning sector and westward in the evening sector. The direction and magnitude of the motion were consistent with those of an auroral substorm. His study suggested that the $\mathrm{E} \times \mathrm{B}$ drift controls the movement of the flux tube that corresponds to cosmic noise absorption (CNA).

Using a scanning multi-narrow-beam riometer at Syowa Station, Antarctica, Kikuchi and Yamagishi (1990) and Kikuchi et al. (1990) showed that the directions in which a belt-shaped absorption region 30-60 km wide drifted were the same as those observed by Hargreaves (1970). Velocity vectors for the drift of the belt-shaped absorption were illustrated by the paper of Kikuchi et al. (1990), which stated that the drift tended to be equatorward in the pre-midnight (2124 MLT) sector and poleward in the pre-noon $(\sim 10 \mathrm{MLT})$ sector.

The spatial and temporal variations of these kinds of events are so great that high-resolution equipment is needed to detect

Copy right (C) The Society of Geomagnetism and Earth, Planetary and Space Sciences (SGEPSS); The Seismological Society of Japan; The Volcanological Society of Japan; The Geodetic Society of Japan; The Japanese Society for Planetary Sciences. them. Therefore, we developed a $16 \times 16$-antenna imaging riometer with very high temporal and spatial resolutions of approximately $1 \mathrm{~s}$ and $10 \mathrm{~km}$, respectively.

Section 2 describes the specifications of this instrument. Section 3 describes the methods used for data reduction and analysis, and Sections 4 and 5 describe and discuss some of the results obtained by observing the drift of the auroral absorption region. Particular attention is paid to the relation of drift to magnetic local time (MLT). Section 6 summarizes this paper.

\section{Instrumentation}

The imaging riometer, which has 256 narrow beams, was constructed in Poker Flat, Alaska $\left(65.1^{\circ} \mathrm{N}, 147.5^{\circ} \mathrm{W}\right.$ in geographic coordinates; $65.4^{\circ} \mathrm{N}, 100.7^{\circ}$ in geomagnetic coordinates) in October 1995 (Murayama et al., 1997). A riometer is a radio telescope that operates in the HF and VHF bands, and is used to measure the intensity of galactic radio noise. Since the source strength of such noise is very stable, temporal variations measured by the riometer reflect noise absorption, which is mainly due to increases in the electron density of the ionospheric D-region. The absorption effects from changes in the D-region electron density can thus be derived by subtracting the observed radio-wave intensity from the quiet-time diurnal variation. The relative absorption is called CNA. A riometer with a broad-beam antenna measures the average absorption in the field of view $(100 \times 100$ $\mathrm{km}$ ), while the 256 narrow beams of the riometer we used provide a two-dimensional image of the absorption in the lower ionosphere (typically assumed to be at a height of 90 $\mathrm{km})$. We used 190 narrow beams out of the total 256, which corresponds to a field of view of $400 \times 400 \mathrm{~km}$ with the best 
spatial resolution (beam separation) of $11 \mathrm{~km}$ found around the zenith, at the assumed height of $90 \mathrm{~km}$. (However, there may be some problems about this estimated spatial resolution as mentioned in the next section.) Furthermore, because the data is recorded every second, the system detects twodimensional motions and deformations of auroral arcs with widths of tens of kilometers.

To reduce artificial radio interference as much as possible, we selected an operating frequency of $38.2 \mathrm{MHz}$ in the astronomical protected band between 37.75 and $38.25 \mathrm{MHz}$. To form the 256 narrow beams, we used a phased array antenna consisting of 256 half-wavelength crossed dipoles arranged in a $61.6 \times 61.6 \mathrm{~m}$ square whose sides were along the geomagnetic latitude and longitude. Each crossed-dipole (turnstile) antenna consisted of two half-wavelength dipole elements (3.5 $\mathrm{m}$ long), and the antenna height was $1 / 4$ the wavelength $(1.96 \mathrm{~m})$. The main lobe of the dipole antenna pointed to the zenith, and each pair of crossed dipoles was connected with a $90^{\circ}$-delay line in order to receive right-handed circularly polarized waves.

The phased array antenna formed a main beam in each of the desired directions through a process of antenna signal phasing. We chose the Butler matrix (Butler and Lowe, 1961) as the principal component of the phasing system. The directions of the roughly $6^{\circ}$-wide main beams were separated by about $7^{\circ}$. The dedicated system-control computer switched the selected row, consisting of 16 beams, from the east to the west at 1/16-s intervals. We obtained 256 phasing patterns each second. The riometer had three operation modes: observation, calibration, and AGC, which could be selected with the control computer. The cosmic noise intensity was measured using the observation mode. In the calibration mode, noise signals from a noise generator with eight different noise-temperature values were fed to the receivers. The AGC mode provided a receiver gain adjustment that reduced the gain drift by comparing the feedback output of the detected signal levels with an internal stable reference level.

The control computer was used to adjust the time constant and bandwidth of the receiver. The signal was detected after IF amplification and bandpass filtering, so the intensity of the signal that was actually detected was proportional to the square root of the cosmic noise power that was received. The signal-intensity output was a voltage between 0 and $10 \mathrm{~V}$. Both the phasing system and the receiver set were located inside a waterproof enclosure on the ground in the center of the antenna field (Kainuma et al., 1996).

In the data acquisition system, the receiver outputs were recorded through a 12-bit analog-to-digital converter. The computer in that system obtained the timing signal from the GPS receiver system at the site and recorded the GPS time together with the data every second. The computer also recorded instrument and environmental temperatures. The riometer and housekeeping data were displayed on the computer monitor and transferred to other computers through local and international digital links. In addition, the control computer accepted requests for parameter changes via the digital links, so we were able to use the computer network to monitor and control the system.

\section{Data Reduction and Analysis}

The riometer signal frequently included various kinds of interference or undesired noise. High-intensity, brief interference noises were removed by simply excluding data whenever the noise intensity was greater than $30 \mathrm{~V}^{2}$ (the cosmic signal intensity is less than $10 \mathrm{~V}^{2}$ ).

We also removed data contaminated by signals from radio stars, which scintillate with a high frequency and significant amplitude. Signals from two major stars, Cas-A and Cyg-A, are often observed by stations in the northern hemisphere. It is easy to identify the signals of radio stars because they move with a steady speed that corresponds to the Earth's rotation.

Radio star signals often appeared in several adjacent beams in a particular east-west (E-W) row, while adjacent beams aligned in the north-south (N-S) direction detected these signals with time lags of a few hours. Radio star signals also appeared in some N-S aligned beams, but less often than in $\mathrm{E}-\mathrm{W}$ rows. The detection of radio star signals by multiple beams could be attributed to system factors. We can suggest two possibilities: one scenario is that the very strong signal of radio stars was detected by side lobes, which might cause an apparent increase in signal level even if there is no radio star in the main beam direction. Another possible cause could be riometer receiver channel switching. Sixteen beams in the E-W row are sequentially switched to scan in the $\mathrm{N}-\mathrm{S}$ direction, and the received radio signals in one $\mathrm{E}-\mathrm{W}$ beam row are fed to a receiver unit. The data disturbed by the scintillating signals from radio stars were excluded from the analysis.

The following method was used to determine the drift velocity. A group of time series data sets of ionospheric absorption obtained with magnetic zonal and meridional beam arrays with a center antenna was used to determine each component of the velocity. First, high-frequency noises were removed from the raw data using a "running minimum". That is, the minimum value in a 1-min window was used for the data value and the window was moved continuously. The temporal variation in the intensity of each beam was smoothed with an FFT low-pass filter whose cut-off period was 3 min. After smoothing, all of the local minima and maxima points in the temporal variation that were observed for each beam were identified. Local minima with significantly small values were identified as actual absorption. The averages of the local minima $\left(\overline{A_{\min }}\right)$ and maxima $\left(\overline{A_{\max }}\right)$ were then calculated. When $\overline{A_{\max }}-A_{\min }(i)$ was larger than $C \times\left\{\overline{A_{\max }}-\overline{A_{\min }}\right\}, A_{\min }(i)$ was identified as the event. The constant $C$ was set between 3 and 5 for each event.

Figures 1(a) and 2(a) show the temporal variations in CNA, with the absorption points shown by asterisks using the method. The 16 curves in the upper and lower panels correspond to the east-west and north-south beams with a center beam, respectively. The dotted lines show the baselines of the beams. The distance between the baselines corresponds to the distance between antenna beams projected towards the reference height $(90 \mathrm{~km})$. The zonal and meridional components of the phase velocity of the drift events were determined from the tilt angle of the regression lines, which was calculated using the least squares method for the detected absorption peaks.

Figures 1(b) and 2(b) show the drift velocities calculated 

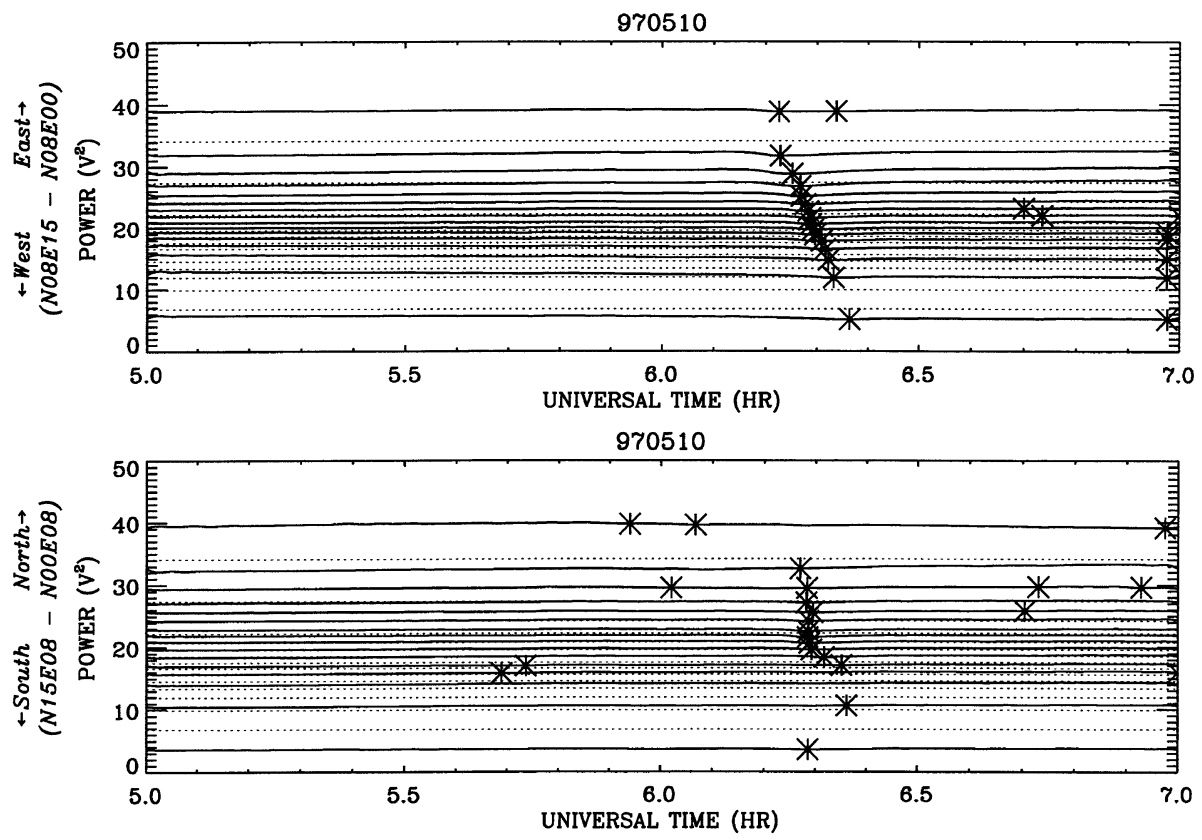

(a)
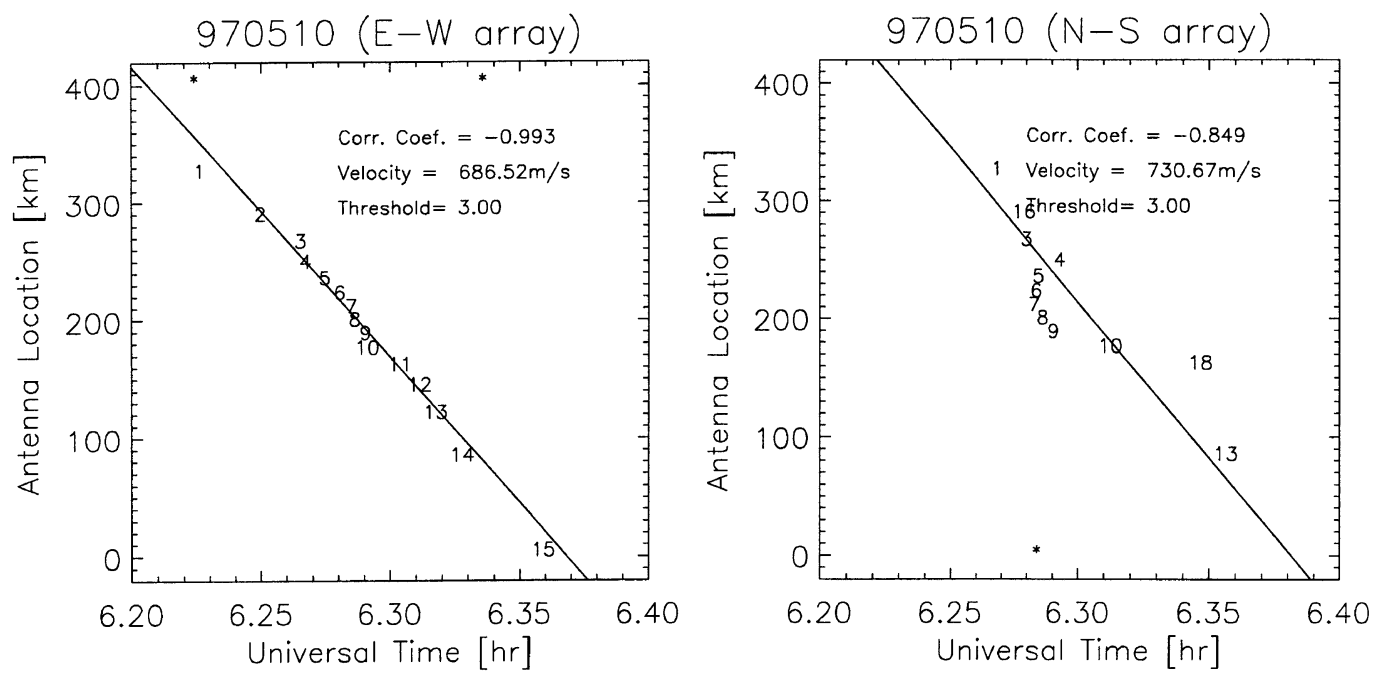

(b)

Fig. 1. Example of a CNA drift event observed in the evening sector (06:10-06:20 UT, 18:00 MLT) on May 10, 1997. (a) Zonal (upper panel) and meridional (lower panel) scan plots obtained with beam arrays with center antenna. The dotted lines are baselines of the beams. The asterisks show automatically detected absorption peaks. The method used to detect absorption is described in the text. (b) Regression lines for absorption peaks shown in (a). The numbers along the line show the absorption peaks used for fitting. Correlation coefficients and velocities are shown in the panels. The meaning of "threshold" is described in the text.

from the tilt angles of the regression lines. The velocity was assumed to be constant when the event was in the field of view (FOV). Some local minimum points unrelated to the drift events inevitably appeared despite the fact that the constant $C$ or other parameters were carefully set. Thus, we had to manually pick the corresponding points after completing the analysis described above. The numbers in Figs. 1(b) and 2(b) represent the selected points, and the asterisks show the other points. The correlation coefficients and estimated velocity components are also shown in the figures. The constant $C$, which was described above, is denoted in the figures as the
"Threshold". The correlation will be discussed in a latter section.

If the absorption region is small enough to be described as a "patch", the vector velocity can be estimated to be

$$
\left|V_{x y}\right|=\sqrt{V_{x}^{2}+V_{y}^{2}},
$$

where $V_{x}$ and $V_{y}$ are the magnetic zonal and meridional components of the velocity, respectively. However, when the structure of an ionospheric absorption region is arc-like, it is difficult to detect velocity components along the arc. Thus, 

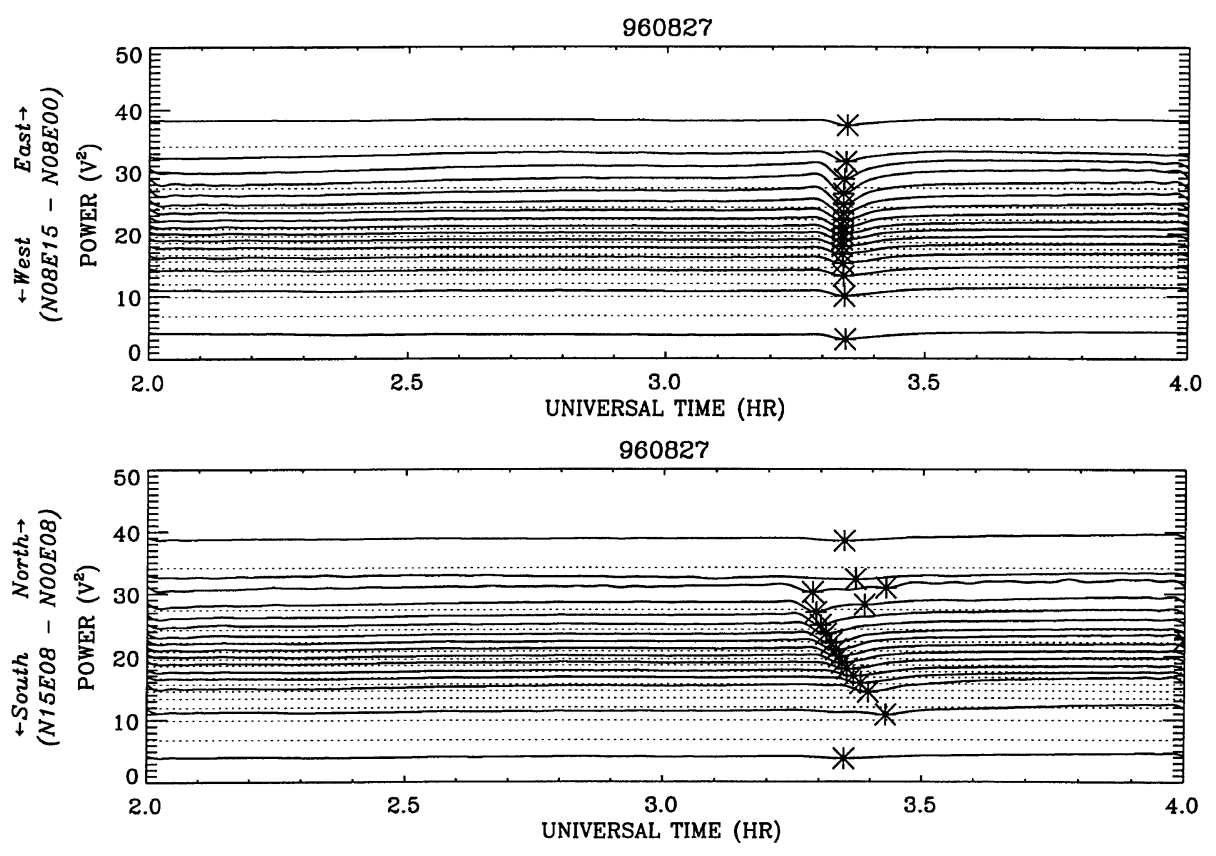

(a)
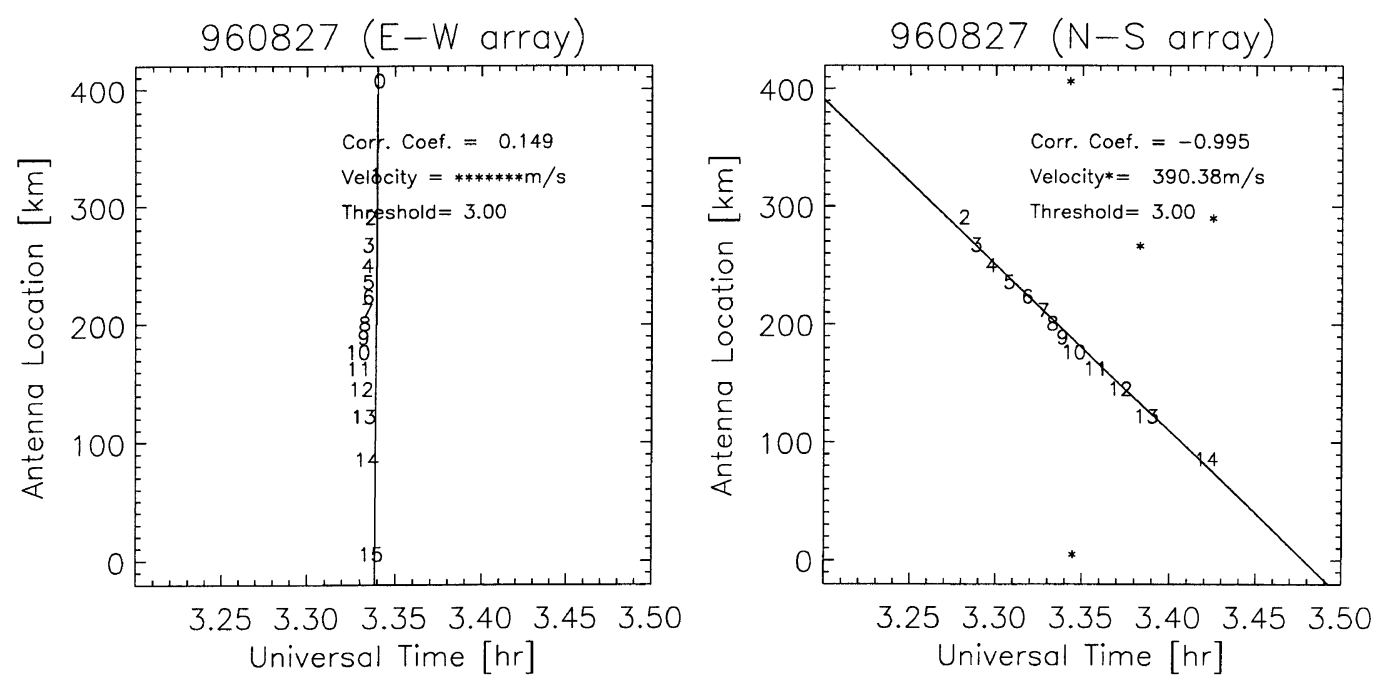

(b)

Fig. 2. Example of a CNA drift event observed in the afternoon sector on August 27, 1996. The explanation is the same as in Fig. 1.

the vector velocity of absorption was determined along a line perpendicular to the arc. Figure 3 is a schematic plot for the configuration of the velocity in case of the arc-like absorption. The zonal and meridional component deduced from absorption on the E-W and N-S rows are $V_{x}$ and $V_{y}$, respectively. The actual drift velocity of arc-like absorption, $V_{d}$, and the tilt angle of the vector velocity, $\theta$, are defined as follows;

$$
V_{d}=V_{y} \cos \theta
$$

where,

$$
\theta=\tan ^{-1}\left(\frac{V_{y}}{V_{x}}\right) .
$$

And the zonal and meridional component of the actual ve- locity, $V_{d x}$ and $V_{d y}$ can be described as;

$$
\begin{gathered}
V_{d x}=V_{d} \cos \theta=V_{x} \cos ^{2} \theta \\
V_{d y}=V_{d} \sin \theta=V_{x} \cos \theta \sin \theta .
\end{gathered}
$$

It should be noted that the zonal and meridional component of $V_{d}$ is generally differenct from those of $V_{x y}$. In this study, the drift absorption velocity was determined using only two scans, zonal and meridional, including the center. This means that the drift velocities of small-scale patches can probably not be derived by the methods used here, because a small drifting patch would give absorption in only one beam in each row or column of beams. Thus, all the cases we discuss here are for arc-like structure, and the drift 


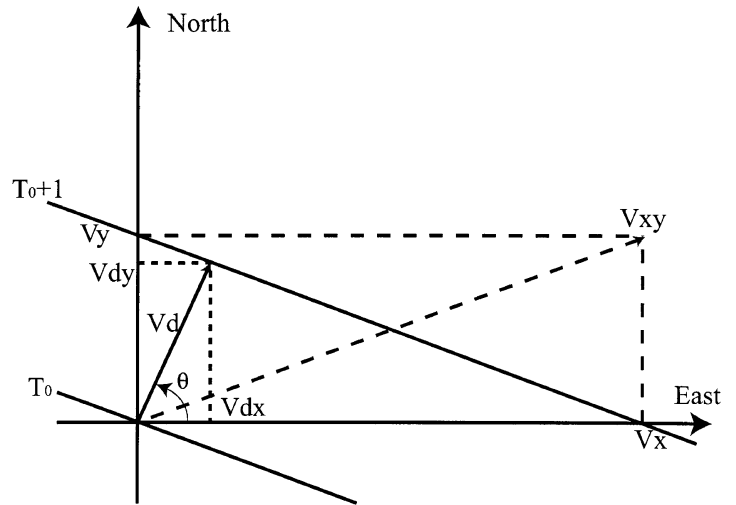

Fig. 3. Configuration of the velocity of arc-like absorption. In this sketch The positions of a moving absorption arc at $T_{0}$ (as the arc crosses the center of the field) and at $T_{0}+1 \mathrm{sec}$ (for convenience). When the zonal and meridional component were observed as $V_{x}$ and $V_{y}$, respectively, the actual drift velocity is defined as $V_{d}$.

velocities are determined by Eq. (2). In addition, structures that passed through the FOV could have been missed if they had not passed through either of the scans.

In all cases, the distributions of the absorption region were checked against a two-dimensional plot of the 256 beams to confirm the shape of the structure.

\section{Observational Results}

\subsection{Drift of cosmic noise absorption}

Figure 1 shows an example of a drift event observed from 06:10 UT to 06:20 UT (18 MLT) on May 10, 1997. The geomagnetic condition was very quiet $\left(\mathrm{Kp}=1_{+}\right)$, and the signal level decreased by approximately $1 V^{2}$ during the event. The drift tendency in the zonal component $\left(V_{x}\right)$ was quite linear, while that in the meridional component $\left(V_{y}\right)$ changed velocity with time. This causes a poor correlation for line fitting with the meridional component $\left(V_{y}\right)$ (Fig. 1(b)). The zonal and meridional components of velocity $\left(V_{x}, V_{y}\right)$ were estimated to be 687 and $731 \mathrm{~m} / \mathrm{s}$, respectively, and the vector velocity $\left(V_{d}\right)$ was calculated to be $\sim 500 \mathrm{~m} / \mathrm{s}$.

Another event, observed from 03:15 to 03:28 UT (15.5 MLT) on August 27, 1996, is shown in Fig. 2. The geomagnetic condition was moderately disturbed $\left(\mathrm{Kp}=4_{-}\right)$, and the signal level decreased by approximately $5 V^{2}$. In the zonal component $\left(V_{x}\right)$, absorption was observed almost simultaneously in all beam rows. The drift tendency in the meridian component $\left(V_{y}\right)$ was quite linear. Fig. 2(b) shows that the zonal component $\left(V_{x}\right)$ of the drift velocity was very large, i.e., the regression line is almost vertical. In this case the correlation is poor because the random fluctuations were large in comparison to the systematic varation. The arc-like structure was found to move southward at $\sim 390 \mathrm{~m} / \mathrm{s}\left(V_{d}\right)$, which is almost the same velocity as that of the meridian component $\left(V_{y}\right)$ of the drift velocity.

\subsection{Local time features of CNA drift}

From October 1, 1995 to October 31, 1997, a total of 106 CNA drift events were identified.

Figure 4 shows the MLT dependence of the directions and magnitudes of the velocity of the absorption events. The arrow colors correspond to the absolute values of the correlation. There are two correlation coefficients for each event, for the zonal and meridional components $\left(V_{x}, V_{y}\right)$; the smaller values are shown here. When the angle between the velocity vector and the cardinal directions (Magnetic North, South,

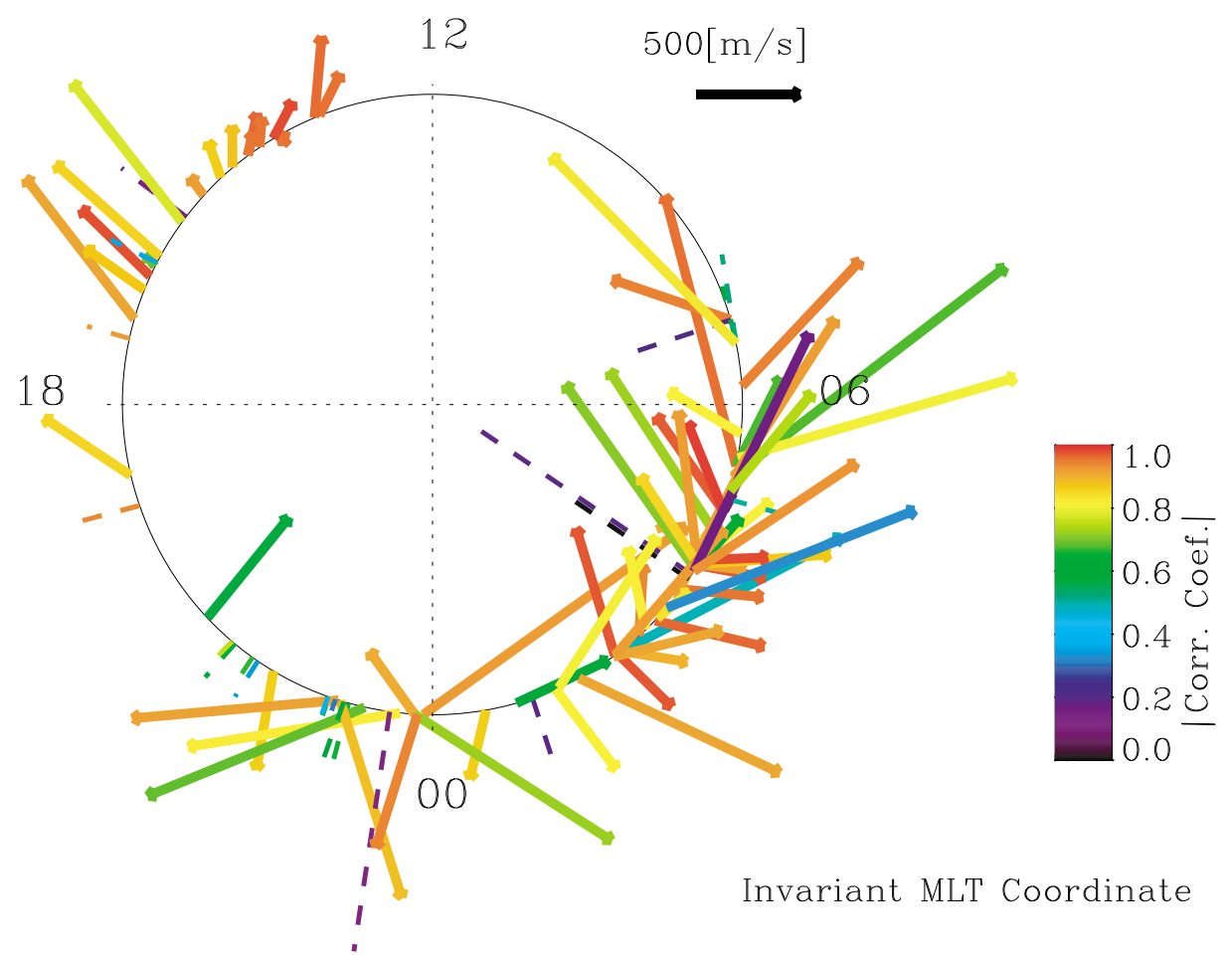

Fig. 4. Polar plot of vector velocities of CNA drifts as a function of MLT. The color of each arrow shows the correlation coefficient used for fitting the regression line. 

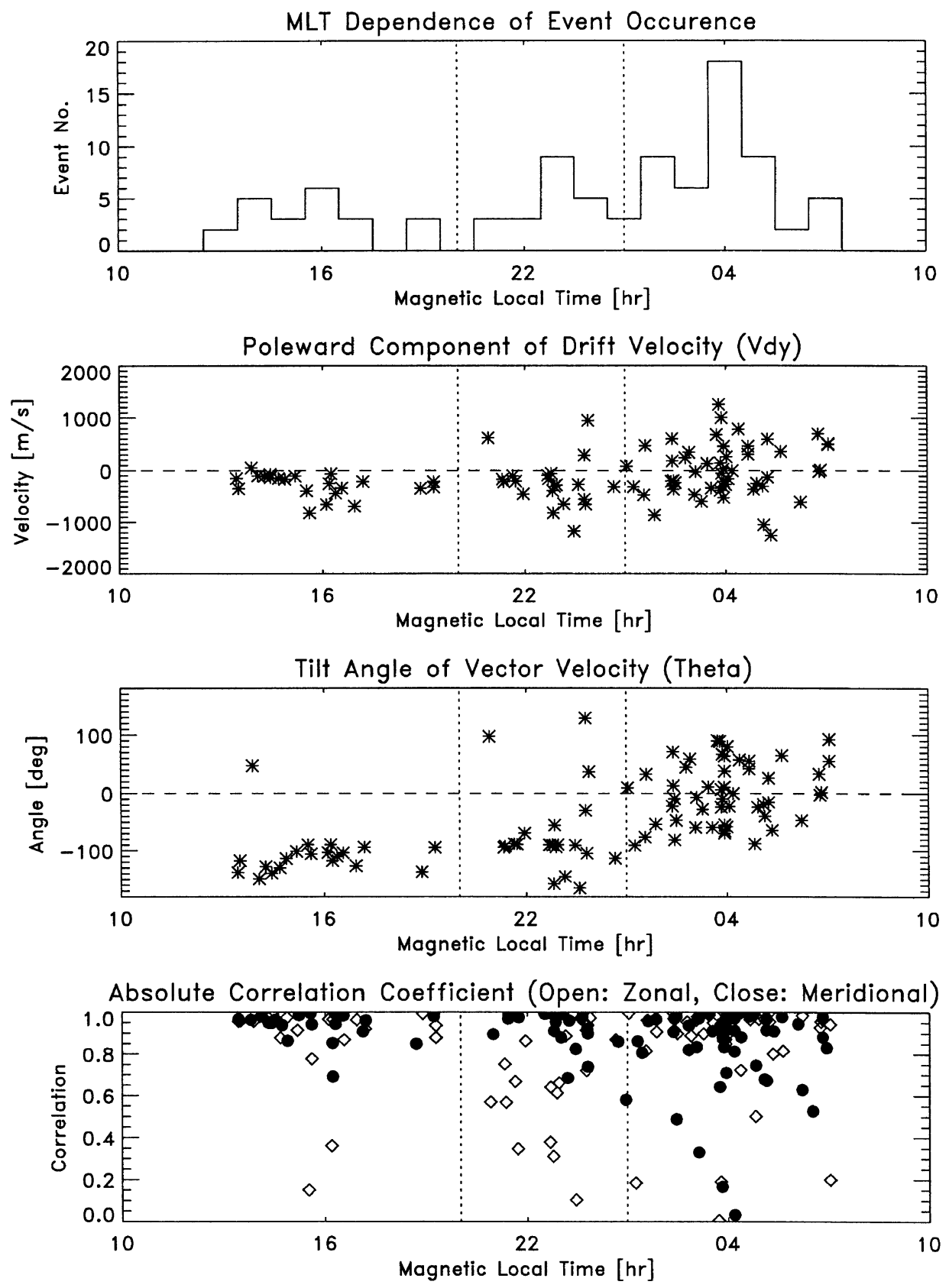

Fig. 5. Top panel: Local time distribution of the number of occurrences of CNA drifts. The total number of events was 106 , and they were observed from October 1995 to October 1997. Second panel: Meridional component of drift velocity (poleward positive). Third panel: Tilt angle, $\theta$, of the arc structure $\left(\theta=\tan ^{-1}\left(V_{y} / V_{x}\right)\right)$, where $V_{x}$ and $V_{y}$ represent the zonal and meridional components of the velocities. Bottom panel: Absolute values of correlation coefficients for each event. Open squares and closed circles show the values for zonal and meridional components of velocities, respectively. The dotted vertical lines show the boundaries between each group, at 20 and 01 MLT.

East and West) is less than $5 \mathrm{deg}$, the vector is shown with broken lines in Fig. 4. The reason of this distinction and the meaning of the correlation coefficient will be discussed in the next section. Although the figure is complicated, some clear tendencies can be seen. To make it easier to see them, the MLT dependences of four parameters are plotted in Fig. 5. From top to bottom they are: event occurrence, meridional component of drift velocity $\left(V_{d y}\right)$, tilt angle of drift velocity vectors $(\theta)$, and absolute value of correlation coefficients. Northward velocity was positive in the meridional component. The tilt angle is the angle between the velocity vector and the eastward axis, and is anti-clockwise positive. The following results are shown in Figs. 4 and 5.

1) From the distribution of event occurrences, the data set can be divided into three groups based on the MLT. Events in the first group were observed between 13 and 19 MLT (group I); those in the second group were observed between 21 and 01 MLT (group II); those in the third group were observed between 01 and 07 MLT (group III). The dotted vertical lines in Fig. 5 show the boundaries between the groups, at 20 and 01 MLT. The occurrence rates for these groups were $20.8 \%, 24.5 \%$, 


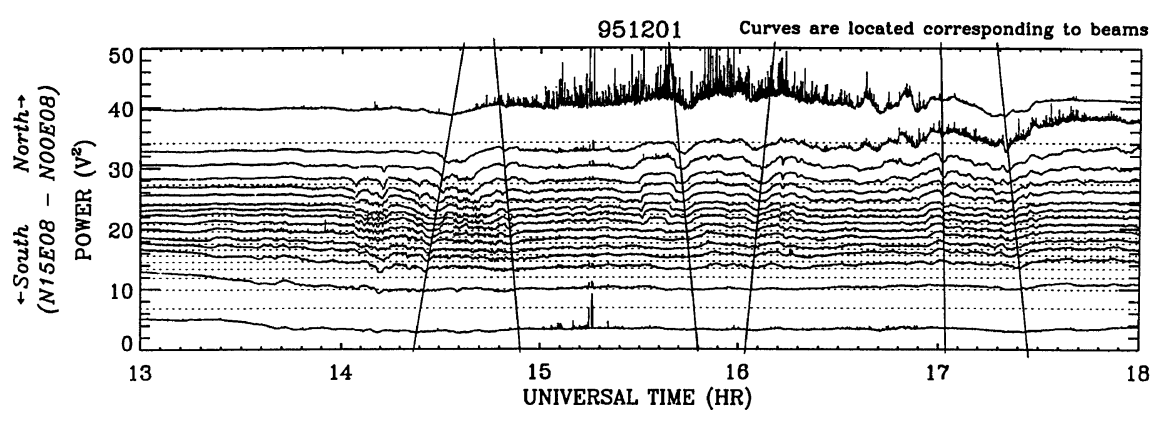

Fig. 6. Example of alternating poleward and equatorward drifts observed on December 01, 1995; meridional scan plot of CNA drift with fitting lines. The directions and velocities of the meridional component of drift velocities are listed in Table 1.

and $54.7 \%$, respectively. The highest occurrence rate (22 occurences) was at approximately 04 MLT, which is in group III.

2) Almost all events in group I moved southwestward with absolute velocities of $\sim 500-1000 \mathrm{~m} / \mathrm{s}$, which is less than the absolute velocities of events in the other groups. The correlations were significantly high in comparison to those of the other groups.

3) The events in group II had various absolute velocities and directions. The correlations greatly varied from case to case.

4) The events in group III also had various absolute velocities. However, the tilt angles were between $-90^{\circ}$ and $90^{\circ}$, which means that all events had eastward positive components. The distribution of correlations was widely spread, but many cases showed a high correlation.

5) Drift events were not found between 07 and 13 MLT in this dataset.

\section{Discussion}

The correlation can be interpreted as follows: There are two cases when the correlation of line fitting became poor: (1) when the arc structure was located almost exactly northsouth or east-west, and (2) when the velocity was not constant. The event presented with broken line in Fig. 4 are in the former category. Most of the events in group I (13-19 MLT) had high correlation, i.e., they moved with constant velocity for both components $\left(V_{x}, V_{y}\right)$. In addition, all events except for one in group I moved equatorward, which is statistically meaningful. However, some events in groups II (21-01 MLT) and III (01-07 MLT) showed low correlation, which did not come from the drift direction. This means that those events changed their drift velocity with time. The clear difference between groups II and III is the distribution of the tilt angle of the vector velocity (third panel of Fig. 5). While there are no tendencies in group II, most of the events in group III had tilt angles between $-90^{\circ}$ and $90^{\circ}$, which means that they were moving eastward.

CNA drift events have been analyzed in previous studies, such as Hargreaves (1970) and Kikuchi et al. (1990), and our results are generally consistent with these results. The
Table 1. Data on the meridional component of drift velocities observed from 14:30 to 17:30 UT, December 05, 1995.

\begin{tabular}{ccc}
\hline Time $(\mathrm{UT})$ & Direction & Meridian velocity $\left(V_{y}\right)$ \\
\hline $14: 30: 00$ & Northward & $560 \mathrm{~m} / \mathrm{s}$ \\
$14: 48: 30$ & Southward & $1100 \mathrm{~m} / \mathrm{s}$ \\
$15: 42: 30$ & Southward & $1340 \mathrm{~m} / \mathrm{s}$ \\
$16: 06: 00$ & Northward & $1020 \mathrm{~m} / \mathrm{s}$ \\
$16: 54: 00$ & Southward & $1200 \mathrm{~m} / \mathrm{s}$ \\
$17: 24: 00$ & Southward & $1110 \mathrm{~m} / \mathrm{s}$ \\
\hline
\end{tabular}

better spatial and temporal resolutions of our observations reduce the uncertainties that arose in the previous studies. The results shown here support those of the previous studies.

A number of equatorward drift events observed between 18 and 24 MLT are found in our results, whereas only four such events were reported by Kikuchi et al. (1990). The results in Hargreaves (1970) showed that in addition to the equatorward component in this region there is a significantly large westward component. A possible reason for the difference is that we mainly looked for arc-like structures, and it is difficult to estimate the velocity component along an arc. We did not find events that had a large westward component like the one found at approximately 02 MLT by Hargreaves (1970).

Events in group II showed no significant tendencies in their velocities, tilt angles, or correlations. It is important to consider where these irregular distributions came from: were they present in one case, or were they the result of the overlapping of different situations? Figure 6 is a meridian scan plot of one event, which may answer these questions. Poleward and equatorward drifts alternately occurred from 14:30 to 17:00 UT (02-05 MLT). The velocities and directions of propagation deduced from the fitting lines are listed in Table 1. Kikuchi and Yamagishi (1990) also described a similar event (see figure 8 in their paper), but it occurred in the pre-noon sector.

A possible explanation for these events is that there were wave-like structures in the absorption region. Studies have shown that auroras have structures, such as omega bands, in the morning sector that are relatively complicated compared to structures in the evening sector (Akasofu, 1981; Oguti, 
1981). Other studies have shown that in many cases the shape of a CNA region corresponds to the shape of the optical aurora (Stoker et al., 1996, 1997). Thus, it is reasonable to conclude that CNA regions in the morning sector often have complicated features (e.g., a wave-like structure). We would like to have studied this inference by comparing the CNA features with the features of the optical aurora, but the allsky camera and meridian scanning photometer at Poker Flat were not available for use in this study.

Some previous studies have shown a plasma convection pattern projected from the magnetosphere to the ionosphere (e.g., Nishida, 1968, 1978). The polar plot of our drift pattern (Fig. 4) had a pattern with features similar to that of convection, equatorward in the pre-midnight sector and sunward in the morning and evening sectors. Hargreaves (1970) suggested that the source region of CNA which is connected by magnetic field line moves with magnetospheric convection. The relation between CNA drift events and magnetospheric convection may become clear by sorting our data set with IMF obtained by satellite observations (e.g., ACE, WIND, etc.). This will be our next step.

\section{Summary}

The 256-beam imaging riometer installed at Poker Flat, Alaska in October 1995 was used to observe cosmic radio noise absorption with high spatial and temporal resolution. Events in which arc-like CNA regions drifted were often observed by this riometer, and we conducted some case studies and statistical analyses of the events observed between October 1995 and October 1997. Statistical results showed that these events can be grouped according to MLT: group I, 13-19 MLT; group II, 21-01 MLT; and group III, 01-07 MLT. Events in group I, which account for $20.8 \%$ of all events, showed southwestward movement with constant velocity. The meridional component of the velocity was found to be as high as $1000 \mathrm{~m} / \mathrm{s}$. The angle of the velocity vector from the eastward axis was between -130 and $-180^{\circ}$. Events in group II (24.5\% of all events) did not show any clear tendencies in their velocities and drift directions. The velocity range of the meridional component was $\pm 1000 \mathrm{~m} / \mathrm{s}$. The range of the velocity vector angle was between -180 and $180^{\circ}$. Events in group III (54.7\% of all events) also showed various velocities, but almost all events showed eastward drift. The range of the velocity vector angle was restricted between -90 and $90^{\circ}$, and the velocity range of the meridional component was similar to that of Event II $( \pm 1000 \mathrm{~m} / \mathrm{s})$. In group II, drift events alternated between poleward and equatorward, showing that the distribution of velocities and drift directions were irregular in group II.

These results are consistent with those of Hargreaves (1970) and Kikuchi et al. (1990), and the high temporal and spatial resolution found in our data confirms the findings in previous studies.
Acknowledgments. We thank Don Rice and June Pelehowski for their support in the operation of the imaging riometer and the observatory at Poker Flat. This study was supported in part by the U.S.-Japan International Research Project to observe the middle atmosphere, CRL, the Ministry of Posts and Telecommunications, Japan. We are very much indebted to our anonymous reviewers for their careful reading of the manuscript and their valuable comments. The editor thanks P. Stauning and E. Nielsen for their assistance in evaluating this paper.

\section{References}

Akasofu, S.-I., Auroral arcs and auroral potential structure, in Physics of Auroral Arc Formation, edited by S.-I. Akasofu and J. R. Kan, pp. 1-14, American Geophysical Union, Washington, D.C., 1981.

Berkey, F. T., V. M. Driatskiy, K. Henriksen, B. Hultqvist, D. H. Jelly, T. I. Shchuka, A. Theander, and J. Yliniemi, A synoptic investigation of particle precipitation dynamics for 60 substorms in IQSY (1964-1965) and IASY(1969), Planet. Space Sci., 22, 255-307, 1974.

Butler, J. and R. Lowe, Beam-forming matrix simplifies design of electronically scanned antennas, Electron. Des., 12, 170-173, 1961.

Hargreaves, J. K., Auroral motions observed with riometers: Movements between stations widely separated in longitude, J. Atmos. Terr. Phys., 29, 1159-1164, 1967.

Hargreaves, J. K., Conjugate and closely-spaced observations of auroral radio absorption-IV The movement of simple features, Planet. Space Sci., 18, 1691-1705, 1970.

Jelly, D. H., On the morphology of auroral absorption during substorms, Can. J. Phys., 48, 335-345, 1970.

Kainuma, S., Y. Murayama, H. Mori, and K. Igarashi, Development of 256-beam Imaging Riometer, Trans. IEICE, B-II, J79-B-II, 7, 422-425, 1996.

Kikuchi, T. and H. Yamagishi, Drift of auroral absorption observed with a scanning beam riometer at Syowa station, J. Commun. Res. Lab., 37, 1-14, March 1990.

Kikuchi, T., H. Yamagishi, and M. Lester, Drift of auroral absorption due to the magnetospheric convection observed with the scanning narrow beam riometer during Sundial-86, Annales Geophys., 8, 431-439, 1990.

Murayama, Y., H. Mori, S. Kainuma, M. Ishii, I. Nishimuta, K. Igarashi, H. Yamagishi, and M. Nishino, Development of a high-resolution imaging riometer for the middle and atmosphere observation program at Poker Flat, Alaska, J. Atmos. Terr. Phys., 59, 8, 925-937, 1997.

Nishida, A., Coherence of geomagnetic DP2 magnetic fluctuations with interplanetary magnetic variations, J. Geophys. Res., 73, 5549-5559, 1968.

Nishida, A., Geomagnetic diagnosis of the magnetosphere, Springer-Verlag, New York, 1978.

Oguti, T., TV observations of auroral arcs, in Physics of Auroral Arc Formation, edited by S.-I. Akasofu and J. R. Kan, pp. 31-41, American Geophysical Union, Washington, D.C., 1981.

Olson, J. V., G. Rostoker, and G. Olchowy, A study of concurrent riometer and magnetometer variations in the Pc 4-5 pulsation band, J. Geophys. Res., 85, 1695-1702, 1980.

Pudovkin, M. I., O. I. Shumilov, and S. A. Zaitzeva, Dynamics of the zone of corpuscular precipitations, Planet. Space Sci., 16, 881-890, 1968.

Stoker, P. H., M. J. Mathews, and M. W. J. Scourfield, Coordinated measurements of auroral light intensities and riometric radio-wave absorption, Geophys. Res. Lett., 23, 641-644, 1996.

Stoker, P. H., M. J. Mathews, and M. W. J. Scourfield, Cosmic radio noise absorption related to structures in auroral luminosity, J. Geophys. Res., 102, 7439-7447, 1997.

Theander, A., Studies of auroral absorption substorms, Ph.D. Thesis, Kiruna Geophysical Observatory, Report No. 723, 1972.

S. Kainuma (e-mail: kainuma@crl.go.jp), M. Ishii, Y. Murayama, T. Kikuchi, H. Mori, and K. Igarashi 